\title{
Autosomal dominant vitreoretinochoroidopathy
}

INSERM

\section{Source}

INSERM. (1999). Orphanet: an online rare disease and orphan drug data base. Autosomal dominant vitreoretinochoroidopathy. ORPHA:3086

Autosomal dominant vitreoretinochoroidopathy (ADVIRC) is a genetic vitreous-retinal disease characterized by ocular developmental anomalies such as microcornea, a shallow anterior chamber, glaucoma and cataract. Abnormal chorioretinal pigmentation is present, usually lying between the vortex veins and the ora serrata for 360 degrees. 